\title{
钠离子电池正极材料 $\mathrm{P} 2-\mathrm{Na}_{x}\left[\mathrm{Mg}_{0.33} \mathrm{Mn}_{0.67}\right] \mathrm{O}_{2}$ 的电化学活性研究
}

\author{
张晓君 ${ }^{1}$, 李佳乐 ${ }^{1,2}$, 邱吴款 ${ }^{2,3}$, 杨水森 ${ }^{1}$, 刘建军 ${ }^{2,3,4}$
}

(1. 东北电力大学 吉林省生物质清洁转化与高值化利用科技创新中心, 吉林 $132012 ; 2$. 中国科学院 上海硅酸盐 研究所, 高性能陶瓷和超微结构国家重点实验室, 上海 200050; 3. 中国科学院大学 材料科学与光电工程中心, 北 京 100049 ; 4. 中国科学院大学 杭州高等研究院, 化学与材料科学学院, 杭州 310024)

摘 要: 钠离子电池具有成本低廉、原料分布广泛等优点, 是锂离子电池正极材料的最佳替代材料。在具有层状结 构的 $\mathrm{P} 2$ 相 $\mathrm{NaMnO}_{2}$ 正极材料中, 对过渡金属层进行二元固溶可有效提升电极材料的电化学性能。本研究利用库仑 模型构建了 $\mathrm{Mg}$ 离子固溶的 $\mathrm{Na}_{x}\left[\mathrm{Mg}_{0.33} \mathrm{Mn}_{0.67}\right] \mathrm{O}_{2}$ 结构模型。通过第一性原理计算发现, 在钠离子含量小于 0.67 时, $\mathrm{Na}_{x}\left[\mathrm{Mg}_{0.33} \mathrm{Mn}_{0.67}\right] \mathrm{O}_{2}$ 的放电电压达到 $3.0 \mathrm{~V}$ 。电子态密度和电荷布居分析共同表明, $\mathrm{Mg}$ 的固溶激活了 $\mathrm{P} 2$ 相 $\mathrm{Na}_{x}\left[\mathrm{Mg}_{0.33} \mathrm{Mn}_{0.67}\right] \mathrm{O}_{2}$ 中晶格氧的电化学活性, 使体系的电化学反应机制从阴阳离子协同电化学反应转变为可逆阴离 子电化学反应。这一机理为钠离子电池电极材料的设计提供了一种全新方法, 也为其它离子电池的优化和探索提供 了全新的思路。

关 键 词: 钠离子电池; 电化学活性; 第一性原理; 碱金属掺杂

中图分类号: 0646 文献标志码: A

\section{Electrochemical Activity of Positive Electrode Material of P2- $\mathrm{Na}_{x}\left[\mathrm{Mg}_{0.33} \mathrm{Mn}_{0.67}\right] \mathrm{O}_{2}$ Sodium Ion Battery}

\begin{abstract}
ZHANG Xiaojun $^{1}$, LI Jiale ${ }^{1,2}$, QIU Wujie ${ }^{2,3}$, YANG Miaosen ${ }^{1}$, LIU Jianjun ${ }^{2,3,4}$
(1. Jilin Province Sci-Tech Center for Clean Conversion and High-valued Utilization of Biomass, Northeast Electric Power University, Jilin 132012, China; 2. State Key Laboratory of High Performance Ceramics and Superfine Microstructure, Shanghai Institute of Ceramics, Chinese Academy of Sciences, Shanghai 200050, China; 3. Center of Materials Science and Optoelectronics Engineering, University of Chinese Academy of Sciences, Beijing 100049, China; 4. School of Chemistry and Materials Science, Hangzhou Institute for Advanced Study, University of Chinese Academy of Sciences, Hangzhou 310024, China)
\end{abstract}

\begin{abstract}
With the advantages of low cost and wide distribution of raw materials, sodium-ion batteries are considered to be the best alternative materials for lithium-ion battery cathode materials. In the P2-phase $\mathrm{NaMnO}_{2}$ with layered structure, binary solid solution of the transition metal layer can effectively improve the electrochemical performance of the electrode material. In this study, the structural model of $\mathrm{Na}_{x}\left[\mathrm{Mg}_{0.33} \mathrm{Mn}_{0.67}\right] \mathrm{O}_{2}$ with $\mathrm{Mg}$ ion solid solution was constructed by using the Coulombic model. The first-principles calculations revealed that discharge voltage of $\mathrm{Na}_{x}\left[\mathrm{Mg}_{0.33} \mathrm{Mn}_{0.67}\right] \mathrm{O}_{2}$ reached $3.0 \mathrm{~V}$ at a sodium ion content of less than 0.67 . Electronic density of states and charge population analysis showed that the solid solution of $\mathrm{Mg}$ motivated the anionic electrochemical activity
\end{abstract}

收稿日期：2020-09-14；收到修改稿日期：2020-11-13; 网络出版日期：2020-12-11

基金项目：国家自然科学基金(21973107,11804351); 吉林省教育厅项目(JJKH20190693KJ)

National Natural Science Foundation of China (21973107, 11804351); Project of the Education Department of Jinlin Province (JJKH20190693KJ)

作者简介：张晓君(1974-)，女，博士，高级实验师. E-mail: zhangxjun1123@126.com ZHANG Xiaojun(1974-), female, PhD, senior engineer. E-mail: zhangxjun1123@126.com

通信作者：刘建军，研究员. E-mail: jliu@mail.sic.ac.cn LIU Jianjun, professor. E-mail: jliu@mail.sic.ac.cn 
of lattice oxygen in the P2-phase $\mathrm{Na}_{x}\left[\mathrm{Mg}_{0.33} \mathrm{Mn}_{0.67}\right] \mathrm{O}_{2}$, which transformed the electrochemical reaction mechanism of the system from cationic and anionic synergic redox reaction to reversible anionic redox reaction. This transformation provides a novel method for the design of electrode materials for $\mathrm{Na}$ ion batteries, as well as a new approach for the optimization and exploration of other ion batteries.

Key words: sodium ion battery; electrochemical activity; first principle; alkali metal doping

近年来，随着风能、太阳能等新能源产业的市场 化, 人们对大规模储能技术提出了更高的要求 ${ }^{[1-2]}$ 。 在储能材料的研究中, 电极材料的电化学活性和结 构稳定性是研究的焦点之一 ${ }^{[3-4]}$ 。在目前已知的多种 储能体系中, 相对成熟且应用较为广泛的是锂离子 电池材料。然而锂资源分布不均、锂回收技术难等 问题严重制约了锂离子电池的大规模应用 ${ }^{[5-7]}$ 。与锂 元素同族的钠具有原料丰富、成本低廉、分布广泛 等特点。同时, 钠离子电池具有与锂离子电池相似 的物化性质和电化学反应机理。因此, 钠离子电池 是一类最具潜力的替代材料 ${ }^{[8-9]}$ 。

Berthelot 等 ${ }^{[10]}$ 研究发现, 在含单一过渡金属 (TM)的层状氧化物 $\mathrm{NaTMO}_{2}$ 中, 钠离子和空位在纯 钠层中有序排列, 使得该类氧化物在放电过程中存 在诸多电压平台, 导致比容量迅速衰减和循环性能 大幅降低, 因此该类氧化物的能量转换效率较低。 在过渡金属层引入固溶元素, 形成二元甚至多元过 渡金属混排的电极材料中含有大量无序电荷, 有效 地抑制上述电压平台, 提高能量转化效率。Yabuuchi 等 ${ }^{[11]}$ 使用 $\mathrm{Na}_{2} \mathrm{CO}_{3}$ 、 $\left(\mathrm{MgCO}_{3}\right)_{4} \mathrm{Mg}(\mathrm{OH})_{2} \cdot 5 \mathrm{H}_{2} \mathrm{O}$ 和 $\mathrm{MnCO}_{3}$ 为原料, 在 $900{ }^{\circ} \mathrm{C}$ 进行 $12 \mathrm{~h}$ 的固相反应, 获 得 $\mathrm{Mg}$ 固溶的二元无序 $\mathrm{P} 2$ 相 $\mathrm{Na}_{2 / 3}\left[\mathrm{Mg}_{1 / 3} \mathrm{Mn}_{2 / 3}\right] \mathrm{O}_{2}$ 电 极材料。他们发现在 $10 \mathrm{~mA} / \mathrm{g}$ 的电流密度下, 制备 出的 P2 相 $\mathrm{Na}_{2 / 3}\left[\mathrm{Mg}_{1 / 3} \mathrm{Mn}_{2 / 3}\right] \mathrm{O}_{2}$ 正极材料的初始比容 量约为 $150 \mathrm{mAh} / \mathrm{g}^{[11]}$, 稍低于 $\mathrm{Na}_{2 / 3} \mathrm{MnO}_{2}$ 的比容量 $(184 \mathrm{mAh} / \mathrm{g})$ 。Bruce 等 ${ }^{[12]}$ 发现 P2 相 $\mathrm{Na}_{2 / 3}\left[\mathrm{Mg}_{1 / 3} \mathrm{Mn}_{2 / 3}\right] \mathrm{O}_{2}$ 中虽然存在晶格氧的电化学反应, 却未观察到氧气 析出, 表明引入 $\mathrm{Mg}$ 提升了材料的循环可逆性和可 逆比容量。然而, 在充放电过程中, 该体系晶格氧的 微观电化学反应机理仍不清楚, $\mathrm{Mg}$ 固溶对提高体 系的稳定性机理也不清晰。

因此，本工作以 $\mathrm{P} 2$ 相 $\mathrm{Na}_{x}\left[\mathrm{Mg}_{0.33} \mathrm{Mn}_{0.67}\right] \mathrm{O}_{2}$ 为研 究对象, 采用密度泛函理论(DFT)的第一性原理计 算方法, 对 $\mathrm{Mg}$ 离子固溶的 $\mathrm{Na}_{x}\left[\mathrm{Mg}_{0.33} \mathrm{Mn}_{0.67}\right] \mathrm{O}_{2}$ 正极 材料的放电性能进行了系统的电化学活性与结构稳 定性研究, 以期在电子、原子等微观尺度阐明电极 材料在电化学反应中的微观机理, 为电化学过程的 理解和新材料的设计提供参考。

\section{1 计算方法}

本工作的计算基于密度泛函理论的平面波基矢 软件 VASP 软件包 ${ }^{[13-14]}$, 采用缀加平面波方法 ${ }^{[15]}$, 交换关联泛函采用 Perdew-Burker-Ernzerhof 形式的

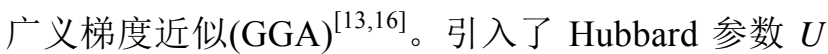
用以修正 $\mathrm{Mn}$ 的 $\mathrm{d}$ 电子, 有效 $U$ 值为 $3.9 \mathrm{eV}^{[17-18]}$ 。 下平面波的截断能为 $600 \mathrm{eV}$ 。当离子弛豫完成时, 所有原子的受力均小于 $0.1 \mathrm{eV} \cdot \mathrm{nm}^{-1}$ 。当优化晶体结 构时, 均采用 $3 \times 3 \times 1$ (72 个原子)的超胞结构, 晶格 常数为 $0.874 \mathrm{~nm} \times 0.874 \mathrm{~nm} \times 1.056 \mathrm{~nm}$, 布里洣区的 $k$ 点网格为 $3 \times 3 \times 3^{[19]}$ 。利用冻声子法在 Phonopy 软件 包中进行了晶格振动谱的计算。为避免周期性边界 条件的影响, 采用 $3 \times 3 \times 1$ 的超胞结构对 $\mathrm{P} 2$ 相 $\mathrm{NaMnO}_{2}$ 和 $\mathrm{Na}\left[\mathrm{Mg}_{0.33} \mathrm{Mn}_{0.67}\right] \mathrm{O}_{2}$ 进行力常数和声子谱 的计算。利用点电荷库仑模型对脱钠结构的离子占 位进行快速计算, 笁选出库仑能最低的 $\mathrm{Na}$ 占据构 型，用以更为精确的第一性原理计算 ${ }^{[20]}$ 。电极材料 的放电电压可表示为 ${ }^{[20]}$ :

$$
V=-\frac{G\left(\mathrm{Na}_{x_{2}} \mathrm{MO}_{2}\right)-G\left(\mathrm{Na}_{x_{1}} \mathrm{MO}_{2}\right)-\left(x_{2}-x_{1}\right) G(\mathrm{Na})}{\left(x_{2}-x_{1}\right) e^{-}}
$$

其中 $G$ 是对应体系的总能量, $e^{- \text {是元电荷 }}{ }^{[21]}$ 。

\section{2 结果和讨论}

\section{1 微观结构特征与结构稳定性}

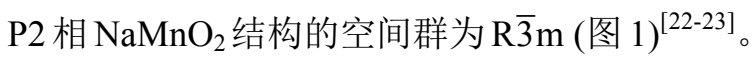
$\mathrm{Mg}$ 固溶 $\mathrm{Na}_{0.67}\left[\mathrm{Mg}_{0.33} \mathrm{Mn}_{0.67}\right] \mathrm{O}_{2}$ 结构的空间构型与 $\mathrm{NaMnO}_{2}$ 类似, $\mathrm{Mg}$ 离子取代了过渡金属层中 $1 / 3$ 的 $\mathrm{Mn}$ 离子, $\mathrm{Mg}$ 与 $\mathrm{Mn}$ 的理论离子比为 $1: 2$ 。实验表 征发现在此比例下, $\mathrm{Na}_{x}\left[\mathrm{Mg}_{0.33} \mathrm{Mn}_{0.67}\right] \mathrm{O}_{2}$ 结构中 $\mathrm{Mg}$ 离子仅与 $\mathrm{Mn}$ 形成无序混排, 保留了 $\mathrm{Na}$ 层的有序 性 ${ }^{[24]}$ 。而当离子比 $\mathrm{Mg}: \mathrm{Mn}>1: 2$ 时, $\mathrm{Mg} 、 \mathrm{Na} 、 \mathrm{Mn}$ 将形成阳离子的无序混排。如图 1(A)所示, 晶格氧 的堆积方式为 $\mathrm{ABBA} \cdots, \mathrm{Mg}$ 和 $\mathrm{Mn}$ 分别占据氧 $\mathrm{AB}$ 层间的八面体位点, $\mathrm{Na}$ 占据氧 $\mathrm{AA}$ 和 $\mathrm{BB}$ 层间的三

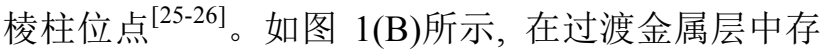
在 $\mathrm{Mg}$ 和 $\mathrm{Mn}$ 的蜂窝状排布 ${ }^{[27]}$, 与富锂化合物中 $\mathrm{Li}$ 

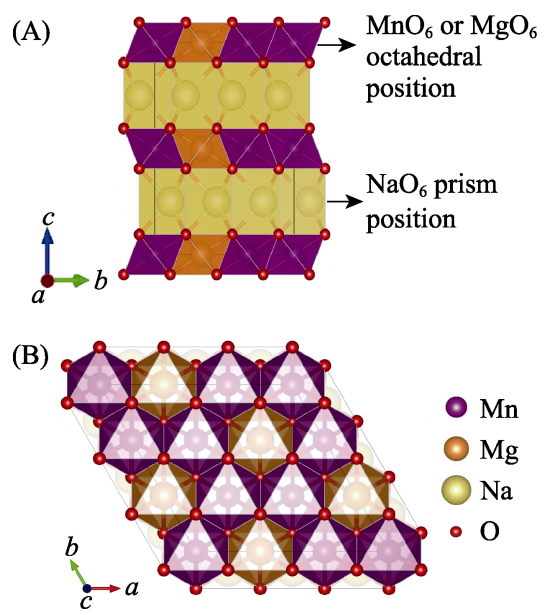

图 $1 \mathrm{P} 2$ 相 $\mathrm{Na}_{2 / 3}\left[\mathrm{Mg}_{1 / 3} \mathrm{Mn}_{2 / 3}\right] \mathrm{O}_{2}$ 结构示意图

Fig. 1 Schematic diagram of $\mathrm{P} 2-\mathrm{Na}_{2 / 3}\left[\mathrm{Mg}_{1 / 3} \mathrm{Mn}_{2 / 3}\right] \mathrm{O}_{2}$

和 $\mathrm{Mn}$ 之间的排列相似 ${ }^{[28]}$ 。 $\left[\mathrm{MgO}_{6}\right]$ 八面体与 6 个 $\left[\mathrm{MnO}_{6}\right]$ 八面体共边排列 ${ }^{[29-30]}$ 。在 $\mathrm{Na}_{0.67}\left[\mathrm{Mg}_{0.33} \mathrm{Mn}_{0.67}\right] \mathrm{O}_{2}$ 结 构的碱金属层中, 钠离子存在两种晶格位点, 一种 与上下两层 $\left[\mathrm{MgO}_{6}\right]$ 或 $\left[\mathrm{MnO}_{6}\right]$ 八面体共边排列, 另一 种与上下两层 $\left[\mathrm{MgO}_{6}\right]$ 或 $\left[\mathrm{MnO}_{6}\right]$ 八面体共面排列。

在不同钠离子含量的结构中, 钠离子受过渡金 属层中 $\mathrm{Mn}$ 和 $\mathrm{Mg}$ 以及碱金属层中 $\mathrm{Na}$ 离子之间的库 仑相互作用, 展现出两种不同的占位方式。因此, 本 工作首先利用库仑模型快速篮选出库仑能最低的 $\mathrm{P} 2$ 相 $\mathrm{Na}_{0.67}\left[\mathrm{Mg}_{0.33} \mathrm{Mn}_{0.67}\right] \mathrm{O}_{2}$ 构型。为了验证合理性, 我们计算模拟了这些笁选构型的 XRD 图谱, 并与实 测结果进行对比 ${ }^{[11]}$, 结果如图 2 所示。计算的(016) 和(110)与实验表征相比稍有右移, 这主要是由于实 验制备的材料在部分晶面中存在非晶和缺陷结构, 而计算模型的结构为完美的晶体结构，因此计算模 拟的 XRD 展宽和峰强与实验结果存在一定偏差。 此外, 这两个晶面中均存在 $\mathrm{Na}$ 离子的排布, $\mathrm{Na}$ 离子 的嵌入与脱出是导致对应峰位偏移的另一可能原 因。考虑上述影响后, 模拟 XRD 的峰型和强度与实

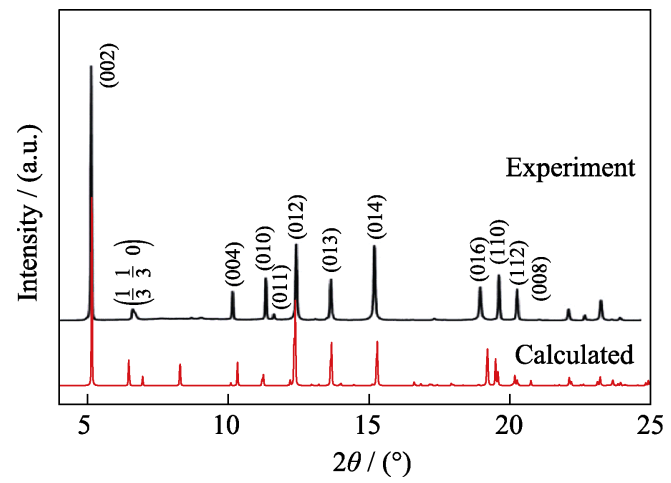

图 $2 \mathrm{P} 2$ 相 $\mathrm{Na}_{0.67}\left[\mathrm{Mg}_{0.33} \mathrm{Mn}_{0.67}\right] \mathrm{O}_{2}$ 模拟与实验 XRD 对比 Fig. 2 Comparison of calculated and experimental XRD patterns of $\mathrm{Na}_{0.67}\left[\mathrm{Mg}_{0.33} \mathrm{Mn}_{0.67}\right] \mathrm{O}_{2}$
验结果吻合, 构建的模型可以复现实验中微观结构 信息, 表明理论篎选的结构较为准确可靠 ${ }^{[31-32] 。}$

为了研究 $\mathrm{Mg}$ 固溶对结构稳定性的影响, 我们 利用第一性原理结合 “冻声子法”分别计算了 P2$\mathrm{NaMnO}_{2}$ 和 $\mathrm{P} 2-\mathrm{Na}\left[\mathrm{Mg}_{0.33} \mathrm{Mn}_{0.67}\right] \mathrm{O}_{2}$ 的晶格振动谱。 如图 3 所示, 所有格波在整个布里渊区内不存在虚 频, 表明 $\mathrm{P} 2-\mathrm{Na}\left[\mathrm{Mg}_{0.33} \mathrm{Mn}_{0.67}\right] \mathrm{O}_{2}$ 具有动力学稳定性。 通过对比两种材料的声子谱发现, $\mathrm{Mg}$ 掺杂未大幅改 变振动频率范围, 对晶格振动的影响较小, $\mathrm{Mg}$ 掺杂 结构也表现出较好的动力学稳定性。此外, Bruce 等 成功制备出 $\mathrm{Mg}$ 固溶的 $\mathrm{P} 2$ 相 $\mathrm{Na}\left[\mathrm{Mg}_{0.33} \mathrm{Mn}_{0.67}\right] \mathrm{O}_{2}$, 也 进一步说明该材料额外具备热力学稳定性。因此, 不 难看出 $\mathrm{P} 2-\mathrm{Na}\left[\mathrm{Mg}_{0.33} \mathrm{Mn}_{0.67}\right] \mathrm{O}_{2}$ 具有很好的结构稳定性。

\section{$2.2 \mathrm{P} 2$ 相 $\mathrm{Na}_{x}\left[\mathrm{Mg}_{1 / 3} \mathrm{Mn}_{2 / 3}\right] \mathrm{O}_{2}$ 电化学性能分析}

为了研究 $\mathrm{Mg}$ 掺杂对材料电化学性能的影响, 我们计算了 $\mathrm{Mg}$ 固溶结构 $\mathrm{P} 2-\mathrm{Na}_{x}\left[\mathrm{Mg}_{0.33} \mathrm{Mn}_{0.67}\right] \mathrm{O}_{2}$ 的 放电电压(图 4), 其中 $\mathrm{Na}$ 离子的浓度范围由实验确 定, 即 $0.11 \leqslant x \leqslant 0.66^{[11]}$ 。图 4(A) 展示了放电过程中的 结构变化, 其对应的电压(图 4(B))主要包含 3.4、2.9 和 $2.1 \mathrm{~V}$ 等三个平台, 预测的理论容量为 $152 \mathrm{mAh} / \mathrm{g}$, 与实验结果基本一致 ${ }^{[11]}$ 。第一性原理计算的放电电压 曲线稍高于实测结果，其主要原因在于第一性原理 计算忽略了实验测定条件的影响，例如电解液、锂
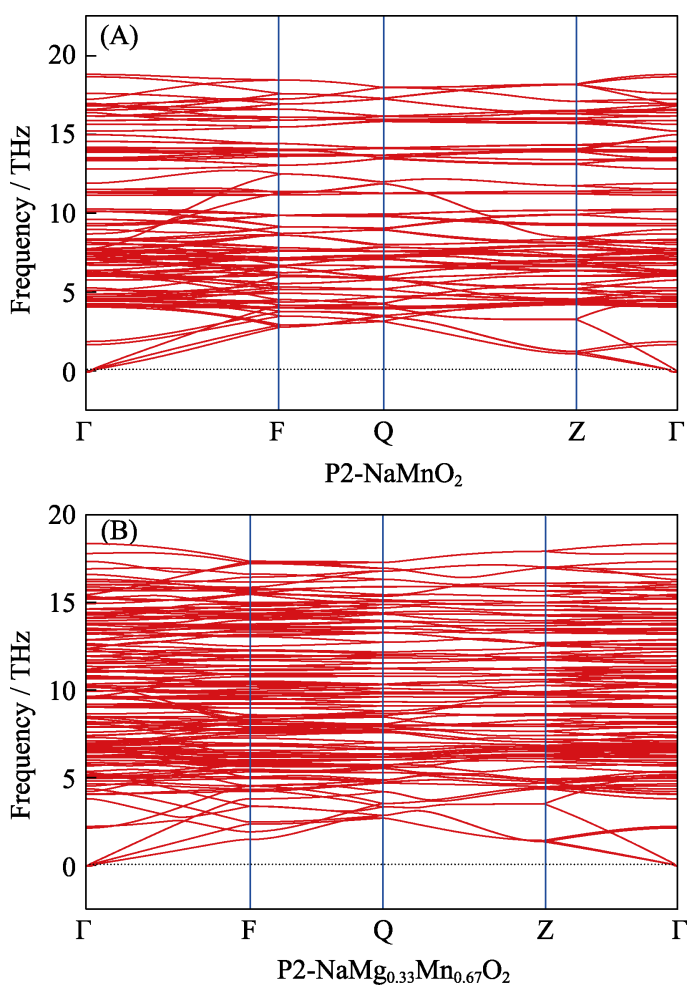

图 $3 \mathrm{P} 2$ 相(A) $\mathrm{NaMnO}_{2}$ 和(B) $\mathrm{Na}_{0.67}\left[\mathrm{Mg}_{0.33} \mathrm{Mn}_{0.67}\right] \mathrm{O}_{2}$ 的晶格 振动谱

Fig. 3 Phonon dispersion curves of (A) $\mathrm{NaMnO}_{2}$ and (B) $\mathrm{Na}_{0.67}\left[\mathrm{Mg}_{0.33} \mathrm{Mn}_{0.67}\right] \mathrm{O}_{2}$ 
$\mathrm{Na}_{0.66}\left[\mathrm{Mg}_{0.33} \mathrm{Mn}_{0.67}\right] \mathrm{O}_{2} \quad \mathrm{Na}_{0.55}\left[\mathrm{Mg}_{0.33} \mathrm{Mn}_{0.67}\right] \mathrm{O}_{2} \quad \mathrm{Na}_{0.44}\left[\mathrm{Mg}_{0.33} \mathrm{Mn}_{0.67}\right] \mathrm{O}_{2}$

(A)

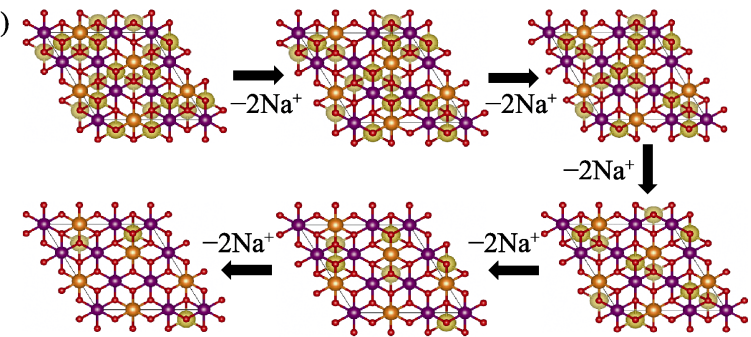

$\mathrm{Na}_{0.11}\left[\mathrm{Mg}_{0.33} \mathrm{Mn}_{0.67}\right] \mathrm{O}_{2} \quad \mathrm{Na}_{0.22}\left[\mathrm{Mg}_{0.33} \mathrm{Mn}_{0.67}\right] \mathrm{O}_{2} \quad \mathrm{Na}_{0.33}\left[\mathrm{Mg}_{0.33} \mathrm{Mn}_{0.67}\right] \mathrm{O}_{2}$ $\circ \mathrm{Mn} \circ \mathrm{Mg} \circ \mathrm{Na} \circ \mathrm{O}$

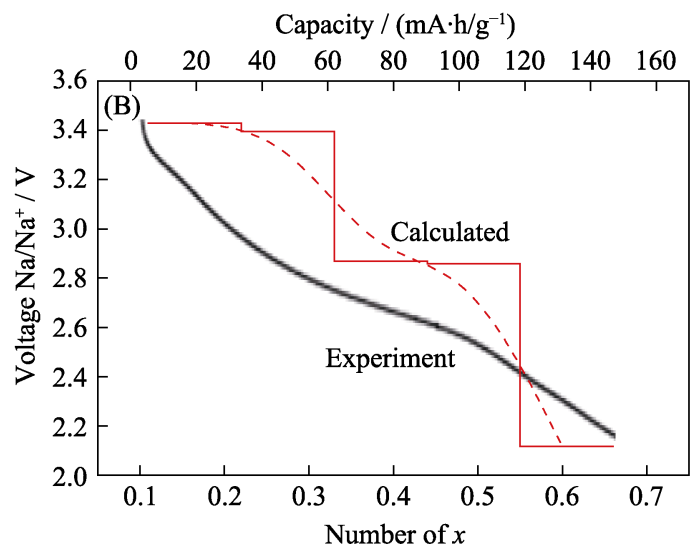

图 4 P2- $\mathrm{Na}_{x}\left[\mathrm{Mg}_{0.33} \mathrm{Mn}_{0.67}\right] \mathrm{O}_{2}$ 的(A)计算得出的放电过程中 结构变化示意图及其 $(\mathrm{B})$ 放电电压曲线。

Fig. 4 (A) DFT-calculated structural changes and (B) discharge voltage curve of $\mathrm{P} 2-\mathrm{Na}_{x}\left[\mathrm{Mg}_{0.33} \mathrm{Mn}_{0.67}\right] \mathrm{O}_{2}$ during discharge

离子电导率实验测定温度等。我们前期研究表明 ${ }^{[33]}$, 虽然计算的放电电压曲线高于实验测定的曲线，但 总体变化趋势是一致的。因此可以认为在整个放电 过程中, $\mathrm{Na}_{x}\left[\mathrm{Mg}_{0.33} \mathrm{Mn}_{0.67}\right] \mathrm{O}_{2}$ 的电压与实验结果较 为吻合 ${ }^{[12,20]}$ 。当 $x<66 \%$ 时, $\mathrm{Na}_{x}\left[\mathrm{Mg}_{0.33} \mathrm{Mn}_{0.67}\right] \mathrm{O}_{2}$ 具有 约 $3.0 \mathrm{~V}$ 的高电压, 且没有出现明显的额外电压平 台, 表明 $\mathrm{Mg}^{2+}$ 取代 $\mathrm{Mn}^{3+}$ 具有抑制钠离子重排以及 结构相变的作用。而此前对 $\mathrm{NaMnO}_{2}$ 等体系的充放 电研究发现, 过渡金属有序排列通常伴随较多的电 压平台。

在理想情况下, $\mathrm{Na}_{2 / 3}\left[\mathrm{Mg}_{0.33} \mathrm{Mn}_{0.67}\right] \mathrm{O}_{2}$ 中 $\mathrm{Mg}$ 和 $\mathrm{Mn}$ 的价态分别为 +2 和 +4 价, 无法继续被氧化至更 高的价态, 因此体系中不存在阳离子电化学活性, 材料的充放电过程为阴离子电化学反应; 而在 $\mathrm{Na}_{0.67} \mathrm{MnO}_{2}$ 中, $\mathrm{Mn}$ 离子的初始价态为 +3.33 , 充电过 程中 $\mathrm{Mn}$ 离子可向外转移 0.67 个电子, 达到稳定的 +4 价, 而此时 $\mathrm{Na}^{+}$已全部脱出, 晶格氧始终不参与电化 学反应 ${ }^{[34]}$ 。因此, $\mathrm{Na}_{0.67} \mathrm{MnO}_{2}$ 的充放电过程表现为阳 离子电化学反应。诸多研究表明, 晶格氧丢失电子 数小于 0.33 时, 阴离子电化学反应具有良好的可逆 性 ${ }^{[11-12,28]}$ 。而氧阴离子的过量氧化(丢失电子数大于 0.33)使氧的电子构型偏离稳定的八耦体规则, 产生不 可逆的转化反应, 形成 $\mathrm{O}-\mathrm{O}$ 键, 甚至导致氧气析出和
电极结构充放电不可逆 ${ }^{[27,35]}$ 。在 $\mathrm{Na}_{0.67}\left[\mathrm{Mg}_{0.33} \mathrm{Mn}_{0.67}\right] \mathrm{O}_{2}$ 中，若考虑电荷丢失的极限状态，即当 $\mathrm{Na}$ 离子完全 脱出形成 $\mathrm{Na}_{0}\left[\mathrm{Mg}_{0.33} \mathrm{Mn}_{0.67}\right] \mathrm{O}_{2}$ 结构、 $\mathrm{Mg}$ 和 $\mathrm{Mn}$ 始终 维持 +2 和 +4 价时, $\mathrm{O}$ 阴离子被氧化至 -1.67 价, 丢失 0.33 个电子, 低于不可逆阴离子电化学反应的界 限。因此, 在 $\mathrm{Na}_{0.67}\left[\mathrm{Mg}_{0.33} \mathrm{Mn}_{0.67}\right] \mathrm{O}_{2}$ 的整个充电反应 中, 晶格氧无需在空间上重组, 其电化学反应具有 可逆性。而引入 $\mathrm{Mg}^{2+}$ 不仅保持了可逆比容量, 还通 过提升放电电压, 额外提升了材料的能量密度。

为了证明 $\mathrm{Na}_{x}\left[\mathrm{Mg}_{0.33} \mathrm{Mn}_{0.67}\right] \mathrm{O}_{2}$ 材料在放电过程 中存在氧的电化学活性, 我们对材料初始和终止放 电结构进行了电子态密度(图 5)计算。结果发现, 在 放电过程中, $\mathrm{Na}$ 离子逐步嵌入, 体系总电子数增加, 费米能级向高能级移动。 $\mathrm{O} 2 \mathrm{p}$ 轨道的空穴数逐渐减 少, 说明进入体系的电子转移到晶格氧的空轨道
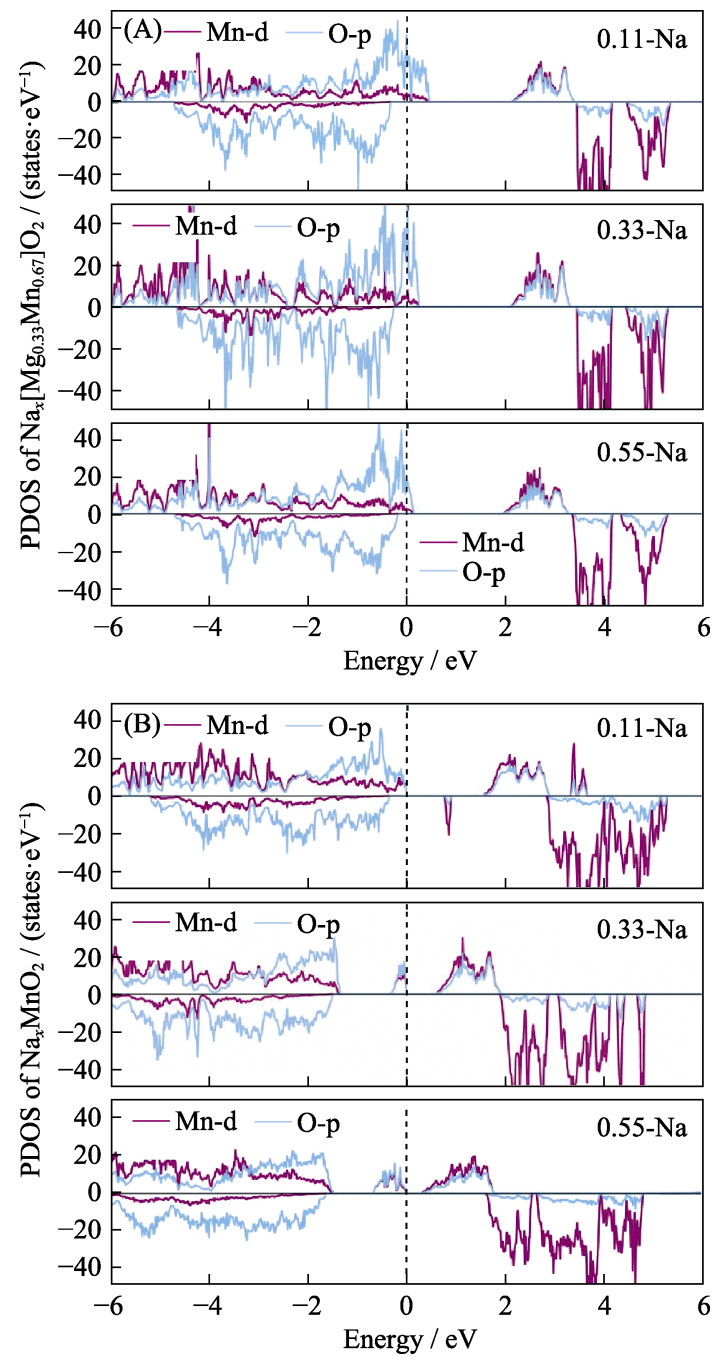

图 5 (A) 不同 $\mathrm{Na}$ 离子含量下的 $\mathrm{P} 2-\mathrm{Na}_{x}\left[\mathrm{Mg}_{0.33} \mathrm{Mn}_{0.67}\right] \mathrm{O}_{2}$ 和 (B) $\mathrm{P} 2-\mathrm{Na}_{x} \mathrm{MnO}_{2}$ 在放电过程中电子态密度

Fig. 5 Electronic density of states of (A) P2- $\mathrm{Na}_{x}\left[\mathrm{Mg}_{0.33} \mathrm{Mn}_{0.67}\right] \mathrm{O}_{2}$ and (B) $\mathrm{P} 2-\mathrm{Na}_{x} \mathrm{MnO}_{2}$ under different $\mathrm{Na}$ ion contents during discharge PDOS: projected density of states 

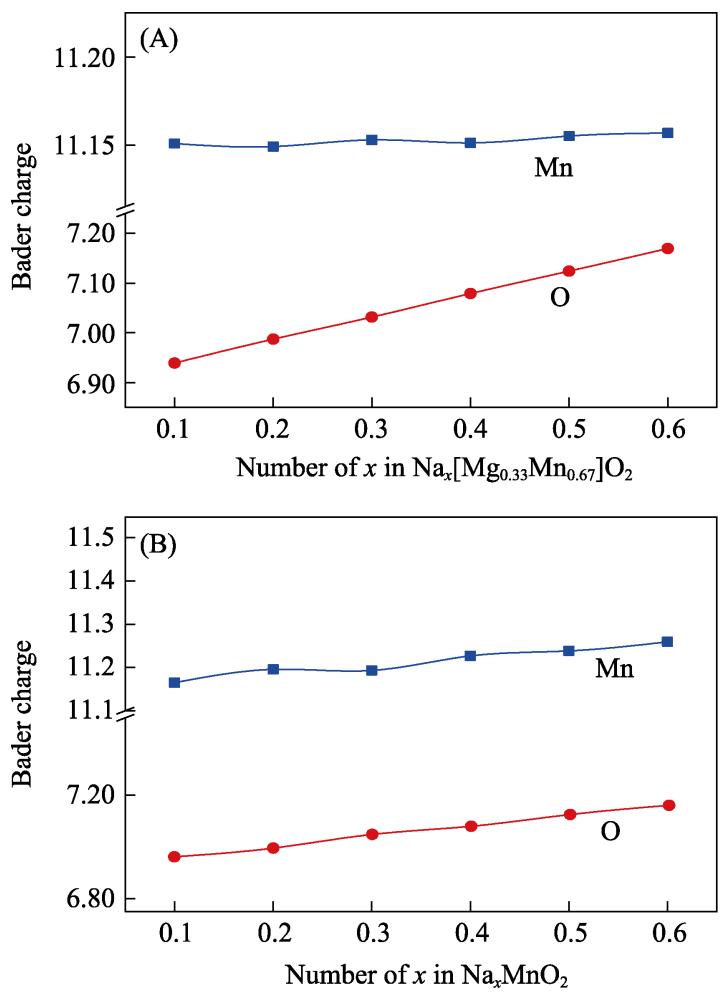

图 6 (A) 在不同钠离子含量下的 $\mathrm{P} 2-\mathrm{Na}_{x}\left[\mathrm{Mg}_{0.33} \mathrm{Mn}_{0.67}\right] \mathrm{O}_{2}$ 和 (B) $\mathrm{P} 2-\mathrm{Na}_{x} \mathrm{MnO}_{2}$ 电荷分析

Fig. 6 Charge analysis of (A) $\mathrm{Na}_{x}\left[\mathrm{Mg}_{0.33} \mathrm{Mn}_{0.67}\right] \mathrm{O}_{2}$ and (B) $\mathrm{P} 2-\mathrm{Na}_{x} \mathrm{MnO}_{2}$ under different sodium ion content

上, 晶格氧被还原, 在电极材料的放电过程中晶格 氧参与了阴离子的电化学反应; 此时, Mn-d 轨道电 子几乎无变化, 不存在电荷转移, 即在放电过程中 $\mathrm{Mn}$ 的价态不发生改变, 证明 $\mathrm{Mn}$ 不具备电化学活 性 ${ }^{[12,36]}$ 。然而在 $\mathrm{P} 2-\mathrm{Na}_{x} \mathrm{MnO}_{2}$ 的放电过程中, 电子不 断向高能级的 $\mathrm{Mn} 、 \mathrm{O}$ 空轨道填充, 说明 $\mathrm{Mn} 、 \mathrm{O}$ 均 具有电化学活性, 是一类典型的阴阳离子协同的电 化学反应。

通过电荷布居分析(图 6)可以得出一致的结论: 在 $\mathrm{Na}_{x}\left[\mathrm{Mg}_{0.33} \mathrm{Mn}_{0.67}\right] \mathrm{O}_{2}$ 的放电过程中, $\mathrm{Mn}$ 离子电荷 量基本不产生变化, 从而不参与电化学反应; $\mathrm{Na}$ 含 量从 0.11 增加到 0.66 的过程中, $\mathrm{O}$ 离子得到了约 $0.2 \mathrm{e}^{-}$, 发生了明显的电荷填充, 表现出了阴离子电 化学活性 ${ }^{[37]}$ 。而通过 $\mathrm{P} 2-\mathrm{Na}_{x} \mathrm{MnO}_{2}$ 的电荷布居分析 发现, 随着 $\mathrm{Na}$ 含量的增加, $\mathrm{Mn}$ 与 $\mathrm{O}$ 共同参与电化 学反应。该结果与电子态密度的分析一致, 证明了 $\mathrm{Mg}$ 的固溶使体系的电化学反应机制从阴阳离子协 同电化学反应转变为可逆阴离子电化学反应, 而这 一过程并不影响材料的充放电可逆性。

\section{3 结论}

本研究利用第一性原理计算系统地研究了 $\mathrm{Mg}^{2+}$
固溶 P2 相 $\mathrm{Na}_{x}\left[\mathrm{Mg}_{1 / 3} \mathrm{Mn}_{2 / 3}\right] \mathrm{O}_{2}$ 的微观结构特征、动 力学稳定性和电化学活性。引入 $\mathrm{Mg}^{2+}$ 使材料的电化 学反应类型从 $\mathrm{Na}_{x} \mathrm{MnO}_{2}$ 的阴阳离子协同电化学反 应转变为 $\mathrm{Na}_{x}\left[\mathrm{Mg}_{0.33} \mathrm{Mn}_{0.67}\right] \mathrm{O}_{2}$ 的可逆阴离子电化学 反应。 $\mathrm{P} 2-\mathrm{Na}_{x}\left[\mathrm{Mg}_{0.33} \mathrm{Mn}_{0.67}\right] \mathrm{O}_{2}$ 中 $\mathrm{O}$ 阴离子参与电化 学反应时, 电荷得失范围小于 0.33 , 具有良好的可 逆性。引入 $\mathrm{Mg}^{2+}$ 不仅提升了材料的放电电压, 而且 保持了材料的可逆比容量, 并最终提升了材料的能 量密度。

在钠离子电极材料中，在过渡金属层引入碱土 金属进行阳离子固溶是一种新的材料性能优化策 略。其基本机理是通过牺牲阳离子电化学活性, 触 发阴离子电化学活性, 改变材料电化学反应机制, 提升放电电压, 并最终优化材料的能量密度。该策 略不仅为钠离子电池电极材料的设计提供了一种全 新方法，也为其它离子电池的优化和探索提供了全 新的思路。

\section{参考文献:}

[1] HU YING-YING, WEN ZHAO-YIN, RUI-KUN, et al. State-ofthe-art research and development status of sodium batteries. Energy Storage Science and Technology, 2013, 2(2): 81-90.

[2] SHEN GUAN-YE, LI CHEN, XU BING-LIANG, et al. Economic allocation for energy storage system considering wind power. Journal of Northeast Electric Power University, 2018, 38(4): 27-34.

[3] MA CHAO, ZHAO XIAO-LIN, KANG LI-TAO, et al. Nonconjugated dicarboxylate anode materials for electrochemical cells. Angew. Chem. Int. Ed., 2018, 57(29): 8865-8870.

[4] RICHARDS W D, DACEK S T, KITCHAEV D A, et al. Fluorination of lithium-excess transition metal oxide cathode materials. Advanced Energy Materials, 2018, 8(5): 1701533.

[5] XIANG XING-DE, ZHANG KAI, CHEN JUN. Recent advances and prospects ofcathode materials for sodium-ion batteries. Adv. Mater., 2015, 27(36): 5343-5364.

[6] MA CHAO, ZHAO XIAO-LIN, HARRIS M M, et al. Uric acid as an electrochemically active compound for sodium-ion batteries: stepwise $\mathrm{Na}^{+}$-storage mechanisms of $\pi$-conjugation and stabilized carbon anion. ACS Applied Materials \& Interfaces, 2017, 9(39): 33934-33940.

[7] LEE D H, XU JING, MENG Y S. An advanced cathode for Na-ion batteries with high rate and excellent structural stability. Phys. Chem. Chem. Phys., 2013, 15(9): 3304-3312.

[8] KUBOTA K, YABUUCHI N, YOSHIDA H, et al. Layered oxides as positive electrode materials for Na-ion batteries. MRS Bulletin, 2014, 39(5): 416-422.

[9] CLÉMENT R J, BRUCE P G, GREY C P. Review-manganesebased P2-type transition metal oxides as sodium-ionbattery cathode materials. Journal of the Electrochemical Society, 2015, 162(14): A2589-A2604

[10] BERTHELOT R, CARLIER D, DELMAS C. Electrochemical investigation of the $\mathrm{P} 2-\mathrm{Na}_{\mathrm{x}} \mathrm{CoO}_{2}$ phase diagram. Nat. Mater., 2011, 10(1): 74-80.

[11] YABUUCHI N, HARA R, KUBOTA K, et al. A new electrode 
material for rechargeable sodium batteries: P2-type $\mathrm{Na}_{2 / 3}\left[\mathrm{Mg}_{0.28} \mathrm{Mn}_{0.72}\right] \mathrm{O}_{2}$ with anomalously high reversible capacity. $J$. Mater. Chem. A, 2014, 2(40): 16851-16855.

[12] MAITRA U, HOUSE R A, SOMERVILLE J W, et al. Oxygen redox chemistry without excess alkali-metal ions in $\mathrm{Na}_{2 / 3}\left[\mathrm{Mg}_{0.28} \mathrm{Mn}_{0.72}\right] \mathrm{O}_{2}$. Nat. Chem., 2018, 10(3): 288-295.

[13] GUO SHAO-HUA, SUN YANG, YI JIN, et al. Understanding sodium-ion diffusion in layered $\mathrm{P} 2$ and $\mathrm{P} 3$ oxides via experiments and first-principles calculations: a bridge between crystal structure and electrochemical performance. NPG Asia Materials, 2016, 8: e266.

[14] JI HUI-WEI, KITCHAEV D A, LUN ZHANG-YAN, et al. Computational investigation and experimental realization of disordered high-capacity Li-ion cathodes based on $\mathrm{Ni}$ redox. Chemistry of Materials, 2019, 31(7): 2431-2442.

[15] LEE J, URBAN A, LI XIN, et al. Unlocking the potential of cation-disordered oxides for rechargeable lithium batteries. Science, 2014, 343(6170): 519-522.

[16] URBAN A, LEE J, CEDER G. The configurational space of rocksalt-type oxides for high-capacity lithium battery electrodes. Advanced Energy Materials, 2014, 4(13): 1400478.

[17] CHAKRABORTY A, DIXIT M, AURBACH D, et al. Predicting accurate cathode properties of layered oxide materials using the SCAN meta-GGA density functional. npj Computational Materials, 2018, 4: 60 .

[18] URBAN A, ABDELLAHI A, DACEK S, et al. Electronic-structure origin of cation disorder in transition-metal oxides. Phys. Rev. Lett., 2017, 119(17): 176402.

[19] ASSAT G, TARASCON J M. Fundamental understanding and practical challenges of anionic redox activity in Li-ion batteries. Nature Energy, 2018, 3(5): 373-386.

[20] YABUUCHI N, NAKAYAMA M, TAKEUCHI M, et al. Origin of stabilization and destabilization in solid-state redox reaction of oxide ions for lithium-ion batteries. Nat. Commun., 2016, 7: 13814.

[21] SANNYAL A, AHN Y, JANG J. First-principles study on the two-dimensional siligene (2D SiGe) as an anode material of an alkali metal ion battery. Computational Materials Science, 2019, 165: 121-128.

[22] LI HONG, HU YONG-SHENG, PAN HUI-LIN, et al. Research progress on electrode material structure of room temperature sodium ion storage battery. Scientia Sinica Chimica, 2014, 44(8): 1269-1279.

[23] WANG YUE-SHENG, XIAO RUI-JUAN, HU YONG-SHENG, et al. $\mathrm{P} 2-\mathrm{Na}_{0.6}\left[\mathrm{Cr}_{0.6} \mathrm{Ti}_{0.4}\right] \mathrm{O}_{2}$ cation-disordered electrode for high-rate symmetric rechargeable sodium-ion batteries. Nat. Commun., 2015,

\section{6: 6954.}

[24] WANG QIN-CHAO, MENG JING-KE, YUE XIN-YANG, et al. Tuning P2-structured cathode material by Na-site Mg substitution for Na-ion batteries. J. Am. Chem. Soc., 2019, 141(2): 840-848.

[25] MENDIBOURD A, DELMAS C, HAGENMULLER C. Electrochemical intercalation and deintercalation of $\mathrm{Na}_{\mathrm{x}} \mathrm{MnO}_{2}$ bronzes. Academic Press, 1985, 57(3): 323-331.

[26] SOMERVILLE J W, SOBKOWIAK A, TAPIA-RUIZ N, et al. Nature of the "Z"-phase in layered Na-ion battery cathodes. Energy \& Environmental Science, 2019, 12(7): 2223-2232.

[27] QU JIE, WANG DONG, YANG ZU-GUANG, et al. Ion-dopingsite-variation-induced composite cathode adjustment: a case study of layer-tunnel $\mathrm{Na}_{0.6} \mathrm{MnO}_{2}$ with $\mathrm{Mg}^{2+}$ doping at $\mathrm{Na} / \mathrm{Mn}$ site. ACS Appl. Mater. Interfaces, 2019, 11(30): 26938-26945.

[28] SATO T, SATO K, ZHAO WEN-WEN, et al. Metastable and nanosize cation-disordered rocksalt-type oxides: revisit of stoichiometric $\mathrm{LiMnO}_{2}$ and $\mathrm{NaMnO}_{2}$. Journal of Materials Chemistry A, 2018, 6(28): 13943-13951.

[29] GUIGNARD M, DELMAS C. Using a battery to synthesize new vanadium oxides. Chemistry Select, 2017, 2(20): 5800-5804.

[30] WANG PENG-FEI, YAO HU-RONG, LIU XIN-YU, et al. $\mathrm{Na}^{+} /$ vacancy disordering promises high-rate $\mathrm{Na}$-ion batteries. Science Advances, 2018, 4(3): eaar6018.

[31] KIM H, KIM D J, SEO D H, et al. Ab initio study of the sodium intercalation and intermediate phases in $\mathrm{Na}_{0.44} \mathrm{MnO}_{2}$ for sodium-ion battery. Chemistry of Materials, 2012, 24(6): 1205-1211.

[32] LI XIN, MA XIAO-HUA, SU DONG, et al. Direct visualization of the Jahn-Teller effect coupled to $\mathrm{Na}$ ordering in $\mathrm{Na}_{5 / 8} \mathrm{MnO}_{2}$. Nat. Mater., 2014, 13(6): 586-592.

[33] WANG YOUWEI, WANG JUNKAI, ZHAO XIAOLIN, et al. Reducing the charge overpotential of $\mathrm{Li}_{-} \mathrm{O}_{2}$ batteries through band-alignment cathode design. Energy \& Environmental Science, 2020, 13(8): 2540-2548.

[34] ZHENG C, RADHAKRISHNAN B, CHU I H, et al. Effects of transition-metal mixing on Na ordering and kinetics inlayered $\mathrm{P} 2$ oxides. Physical Review Applied, 2017, 7(6): 064003.

[35] LUN ZHENG-YAN, OUYANG B, CAI ZI-JIAN, et al. Design principles for high-capacity Mn-based cation-disordered rocksalt cathodes. Chem, 2020, 6(1): 153-168.

[36] SEO D H, LEE J, URBAN A, et al. The structural and chemical origin of the oxygen redox activity in layered and cation-disordered Li-excess cathode materials. Nat. Chem., 2016, 8(7): 692-697.

[37] BAI QIANG, YANG LU-FENG, CHEN HAI-LONG, et al. Computational studies of electrode materials in sodium-ion batteries. Advanced Energy Materials, 2018, 8(17): 1702998. 